\title{
Autoeficacia en estudiantes universitarios: el rol del empoderamiento académico
}

\section{Self-efficacy in university students: the role of academic empowerment}

\author{
Marisa Cecilia Tumino. \\ Universidad Adventista del Plata (Argentina). \\ marisa.tumino@uap.edu.ar \\ Josué Mauricio Quinde. \\ Universidad Adventista del Plata (Argentina). \\ josue.quinde@uap.edu.ar \\ Lilian Noemí Casali. \\ Universidad Adventista del Plata (Argentina). \\ lilian.casali@uap.edu.ar \\ Melissa Raquel Valega. \\ Universidad Adventista del Plata (Argentina). \\ melissa.valega@uap.edu.ar
}

\section{RESUMEN.}

La autoeficacia académica constituye un factor importante para el desempeño óptimo en la universidad. En el actual modelo de enseñanza- aprendizaje, el empoderamiento académico aparece como un proceso que facilita la adquisición de herramientas necesarias para desarrollar la autoeficacia. El empoderamiento del estudiante puede potenciar su accionar para mejorar sus habilidades, adaptarse y transformar su entorno.

El objetivo del estudio fue analizar la relación entre el empoderamiento académico y la autoeficacia de los estudiantes universitarios. Para ello se analizaron los datos de 440 estudiantes de diferentes años y carreras, mediante tres escalas tipo Likert adaptadas al ámbito académico. Se aplicaron correlaciones de Pearson y ecuaciones estructurales (SEM) a las variables estudiadas. Los resultados han mostrado que el Empoderamiento Psicológico desempeña un papel mediador entre el Empoderamiento Estructural y la Autoeficacia. Estos resultados destacan el papel trascendente de las condiciones estructurales que deben sostener el proceso de aprendizaje.

\section{PALABRAS CLAVE.}

Autoeficacia académica, autonomización, empoderamiento académico.

\section{ABSTRACT.}

Academic self-efficacy constitutes an important factor for optimal performance in the university. In the current teaching-learning model, academic empowerment appears as a process that facilitates the acquisition of tools necessary to develop self-efficacy. The empowerment of the student can enhance their actions to improve their skills, adapt and transform their environment.

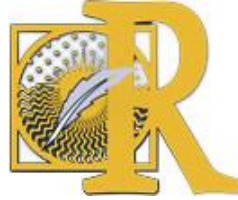

Fecha de recepción: 26-02-2020 Fecha de aceptación: 18-03-2020

Cecilia-Tumino, M., Mauricio-Quinde, J., Noemí-Casali, L. \& Raquel-Valega, M. (2020). Autoeficacia en estudiantes universitarios: el rol del empoderamiento académico International Journal of Educational Research and Innovation (IJERI), 14, 211-224 ISSN: 2386-4303 DOI https://doi.org/10.46661/ijeri.4618 
The objective of the study was to analyze the relationship between academic empowerment and the academic self-efficacy of university students. For this, the data of 440 university students of different years and careers were analyzed, using three Likert-type scales adapted to the academic field. Pearson correlations and structural equations (SEM) were applied to the variables studied. The results have shown that psychological empowerment plays a mediating role between structural empowerment and academic self-efficacy. These results highlight the transcendent role of the structural conditions that must sustain the learning process.

\section{KEY WORDS}

Academic self-efficacy, empowerment, academic empowerment.

\section{Introducción}

El proceso de educación del nivel superior apunta a formar ciudadanos profesionales capacitados para responder a las demandas de la sociedad y actuar con responsabilidad en el medio donde se desenvuelven. Para alcanzar este cometido es necesario que los estudiantes posean cierto nivel de autoeficacia.

Las creencias personales constituyen un factor decisivo para completar tareas, tomar decisiones y alcanzar metas en cada etapa del desarrollo humano. A medida que las creencias de eficacia percibida aumentan, las personas invierten mayor esfuerzo y persistencia para lograr objetivos propuestos (Blanco, 2012). Según Cavazos Arroyo y Encinas Orozco (2016), las creencias de autoeficacia resultarían de suma importancia para el éxito del estudiante; los autores sostienen que la percepción de autoeficacia académica constituye uno de los predictores del engagement académico, lo que repercute directamente en las estrategias de servicio dentro y fuera del aula. Por lo tanto, las instituciones deben atender especialmente las interacciones generadas en el proceso educativo.

La autoeficacia puede conceptualizarse como los juicios de las personas sobre sus capacidades para organizar y ejecutar las acciones necesarias para lograr el desempeño designado (Bandura, 1986, p .391).

Barraza (2010) expone el potencial heurístico de la autoeficacia, manifiesto al constituirse en el objeto de múltiples investigaciones desarrolladas en diferentes ámbitos. En el ámbito académico, parafraseando a Bandura (1987), se puede definir como las creencias de los estudiantes sobre su propia capacidad para realizar las actividades académicas que le son demandadas en su ámbito educativo.

Canto y Rodríguez (1998) estiman que, a partir de esta concepción, para apreciar su autosuficiencia el estudiante valora las percepciones acerca de su capacidad, de la dificultad de una tarea, de la cantidad de esfuerzo involucrado, de la ayuda externa recibida y de las experiencias previas de éxito o de fracaso.

Existen factores que impactan en la autoeficacia de los estudiantes, tal como lo muestra Ventura de La Roca (2012), entre los que se cuentan la confianza y la seguridad que adquieren al aplicar conocimientos o utilizar herramientas, mientras que su percepción varía y se reajusta con cada experiencia que viven. En algunos estudios, Schunk (1991) explicó que la realimentación puede influir en las percepciones sobre la capacidad propia. Estos

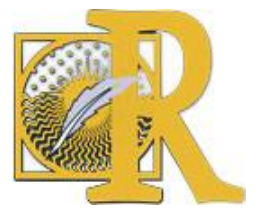


hallazgos podrían suponer que el protagonismo de los estudiantes en su proceso de aprendizaje fortalecería la percepción de autoeficacia.

Desde un abordaje educativo, Pajares (2002) señala que para describir el rol que desempeña la autoeficacia en el comportamiento humano, resulta necesario explorar las cuatro fuentes que originan estas creencias: experiencia de dominio, experiencia vicaria, persuasiones sociales y estados e índices fisiológicos. La experiencia de dominio se adquiere cuando los estudiantes logran mejores resultados y en consecuencia incrementan la percepción de confianza en sus propias capacidades; la experiencia vicaria se logra como efecto producido por el modelado de las acciones de personas significativas de su entorno; las persuasiones sociales se desarrollan en los estudiantes a partir de mensajes de aliento que se reciben de otros que creen en sus capacidades; y los estados fisiológicos cobran importancia puesto que los estudiantes basan su confianza, en parte, en el estado emocional que experimentaron mientras realizaban dicha actividad.

En el contexto planteado, aparece el estudio del empoderamiento como un proceso que facilita la adquisición de las herramientas necesarias para desarrollar la eficacia y eficiencia para alcanzar cualquier meta deseada (Wall, 2012). Con el objeto de conocer el protagonismo de los estudiantes en la percepción de autoeficacia, se incluye en el estudio el concepto de empoderamiento.

La participación e implicación de quienes conforman una organización educativa se convierte en una base para sostener una organización saludable, donde una de las prácticas más conocidas es el empoderamiento organizacional. El empoderamiento no sólo significa poseer autonomía en las decisiones sino también percibir la importancia y el sentido de lo que se realiza (Spreitzer, 1995).

La nueva visión de la educación contrasta con los estilos pedagógicos y evaluación predominantes en el sistema educativo al proponer una educación democrática, incluyente que fortalezca las habilidades, los conocimientos y recursos del que aprende para poder planificar y crear cambios (Magendzo, 2003).

Es de resaltar que el empoderamiento es un fenómeno social. Sánchez (2002), se refiere a este concepto como "un proceso intencional, intersubjetivo y continuo de conversión de los individuos en sujetos conscientes de sí mismos, de las circunstancias y del entorno social, mediante la acción comprensiva, crítica y transformadora sobre sus propias interacciones sociales" (p. 41).

Al abordar el empoderamiento organizacional, los investigadores consideraron dos perspectivas, la perspectiva estructural y la psicológica. La perspectiva estructural parte de la idea de que "el empoderamiento es la transmisión de poder en las organizaciones hacia quienes tienen menos poder" (Jaímez, 2013, p. 24). La perspectiva psicológica hace referencia al proceso de mejora de la percepción de autoeficacia entre las personas del mismo nivel jerárquico. El empoderamiento psicológico desempeña un papel de intermediación entre el rediseño del trabajo y el compromiso organizacional (Chen y Chen, 2008).

De acuerdo con Torres (2009), la educación debe estar inspirada en procesos que promuevan el protagonismo del estudiante no sólo en la construcción del conocimiento, sino en la intervención social, lo que consecuentemente demanda el desarrollo de habilidades de

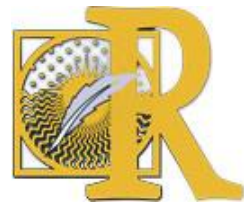


"pensamientos autónomos estructurados reflexivamente con disposición a la crítica y al diálogo" (p. 98). La potenciación de la autoeficacia percibida en quienes aprenden constituye un objetivo educacional valioso, puesto que vehiculiza el logro académico y el desarrollo de metafunciones (Gutiérrez-García y Landeros-Velázquez, 2017). Los resultados armonizan con Pérez (2018) quien entre sus conclusiones sostiene que la autoeficacia académica se muestra como un predictor del rendimiento académico.

En el proceso de enseñanza y aprendizaje, el empoderamiento puede dar cuenta al estudiante de sus capacidades, potenciando su accionar para adaptarse y transformar su entorno. De esta forma, el empoderamiento trasciende lo individual, elevando los niveles de confianza, autoestima y capacidad del estudiante, para atender necesidades propias y colectivas, en un proceso de interacción social (Torres, 2009).

En el presente trabajo se propuso analizar la relación entre el empoderamiento académico y la autoeficacia de los estudiantes universitarios. Específicamente se planteó como objetivo, analizar el empoderamiento psicológico como variable mediadora en la relación entre el empoderamiento estructural y la autoeficacia.

\section{Material y métodos.}

\subsection{Participantes.}

La muestra estuvo compuesta por 440 estudiantes de diferentes carreras universitarias de la región central de Argentina. Un 52\% estuvo representado por mujeres y un 48\% por hombres. El $31 \%$ de los estudiantes pertenecen a la facultad de ciencias sociales, el $31 \%$ de ciencias de la salud, un $21 \%$ de ciencias económicas y administrativas y $17 \%$ de ciencias teológicas. El promedio de edad fue de 22 años con un desvío estándar de 4 años.

\subsection{Instrumentos.}

Las variables propuestas para el estudio fueron autoeficacia y los dos componentes del empoderamiento académico (estructural y psicológico).

\subsubsection{Autoeficacia académica (EAPESA).}

Domínguez et al. (2012) analizaron las propiedades psicométricas de la Escala de Autoeficacia Percibida Específica de Situaciones Académicas (EAPESA) diseñada por Palenzuela (1983) con 10 ítems en una muestra argentina. Para ello evaluaron la fiabilidad (consistencia interna) y validez de constructo (validez de contenido y estructura factorial). El ítem 9 fue eliminado debido a que obtuvo un valor menor de .20 en la correlación ítem-test, mientras que los nueve reactivos restantes obtuvieron valores desde .56 a .73, lo que da cuenta de indicadores satisfactorios de homogeneidad. En cuanto a la validez de contenido, todos los reactivos presentaron intervalos de confianza, correspondientes a la V de Aiken, por encima de .70, lo que brinda evidencias de validez de los reactivos de la escala estudiada. Con relación a la confiabilidad del instrumento, el alfa de Cronbach fue de .89. Respecto de la estructura factorial, Domínguez et al. (2012) corroboraron los hallazgos de Palenzuela (1983) puesto que los nueve reactivos se agruparon en un solo factor que explica el 55.26\% de la varianza total. Se considera que la adecuación de Domínguez et al. (2012), con 9 ítems,

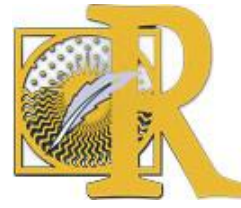


es la apropiada para su aplicación en el contexto donde se desarrolló la presente investigación.

\subsubsection{Empoderamiento académico.}

En el presente trabajo se adaptaron al ámbito académico las escalas propuestas por Jáimez (2013), para medir el empoderamiento organizacional.

El empoderamiento estructural tuvo como base el Conditions of Work Effectiveness Questionnaire (CWEQ) de Laschinger, Finegan, Shamian, and Wilk (2004). La versión en español de la escala (Jaímez y Bretones, 2013) consta de 12 ítems, tres para cada componente del empoderamiento estructural: Apoyo, Recursos, Información y Oportunidades. La escala de respuesta es de tipo Likert de 1 (ninguno) a 5 (mucho). El puntaje total de empoderamiento estructural se obtiene como el promedio de los puntajes de sus ítems. Con respecto a la fiabilidad del CWEQ-II en una muestra Argentina, se encontraron valores satisfactorios del alpha de Cronbach para cada componente: apoyo .804, recursos .769, información .905 y oportunidades .706 (Zambrano y Quinde, 2015).

Para el empoderamiento psicológico se usó la escala de Spreitzer (1995), en versión española (Jáimez, 2013). La estructura interna obtenida en el análisis factorial exploratorio sobre los 13 ítems reflejó un total de 4 factores, que explicaron el $74.08 \%$ de la varianza de la muestra: (a) Competencia, (b) Significado, (c) Autonomía y (d) Impacto. Cada uno de los factores se encuentra compuesto por tres ítems, a excepción de la dimensión Autonomía conformada por cuatro ítems. Los valores del alpha de Cronbach por dimensión son los siguientes: impacto .844, competencia .767, significado .653 y autonomía .831 (Zambrano y Quinde, 2015).

\subsection{Procedimiento de recolección de datos.}

En primer lugar, se contactó con las autoridades universitarias solicitando autorización para realizar la investigación. Posteriormente se consultó a los docentes de cátedra, quienes, en un horario convenido, facilitaron el espacio para evaluar a los estudiantes. Previa explicación verbal de los objetivos de investigación y aclaración de dudas, se invitó a los estudiantes a participar de forma voluntaria, asegurando el tratamiento confidencial y anónimo de los datos. Primero completaron un consentimiento informado escrito y posteriormente los instrumentos. El proceso de evaluación llevó en promedio 20 minutos aproximadamente.

\subsection{Procedimiento de análisis de datos.}

Para realizar el análisis descriptivo de los datos se utilizó el Statistical Package for the Social Sciences (SPSS). Se emplearon cálculos de frecuencia, media, desvío, mínimo, máximo, y correlaciones según el caso.

La estructura de los instrumentos y el análisis de senderos del modelo (path analysis) se aplicaron utilizando el método de máxima verosimilitud en el programa estadístico LISREL en su versión 10.2 (Jöreskog y Sörbom, 2019). Al evaluar el ajuste del modelo, se utilizaron los indicadores de ajuste presentados por Kline (1998), que incluye $\chi 2$, la razón del estadístico chi-cuadrado sobre grados de libertad (X2/df), el error cuadrático medio de aproximación (RMSEA), los índices de bondad de ajuste (GFI y AGFI) y de ajuste comparativo (CFI). Para

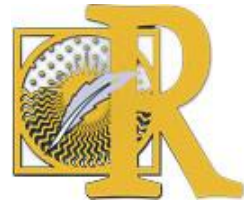


GFI, AGFI y $\mathrm{CFI}$, los valores superiores o iguales a .95 se consideran como indicadores de un buen ajuste del modelo (Hu y Bentler, 1999). Para el RMSEA (Steiger, 1990), se considera que valores inferiores o iguales a .08 son aceptables.

\section{Resultados.}

Como primer paso, se exploró la estructura factorial de la adaptación de los instrumentos de empoderamiento al ámbito académico.

En el caso del Empoderamiento estructural, el instrumento mostró un mejor ajuste al considerar un modelo unidimensional $(\mathrm{X} 2=15.267, \mathrm{DF}=18, \mathrm{p}=.6437, \mathrm{X} 2 / \mathrm{df}=.848, \mathrm{GFI}=$ $.985, \mathrm{AGFI}=.969, \mathrm{CFI}=.999, \mathrm{RMSEA}=.035)$. La escala final quedó compuesta por 8 ítems (ver figura 1), luego de eliminar aquellos que presentaron baja carga factorial $(<.40)$. Se encontró un adecuado nivel de fiabilidad medido a través del coeficiente omega de fiabilidad compuesta (.743) y alfa de Crombach (.689).

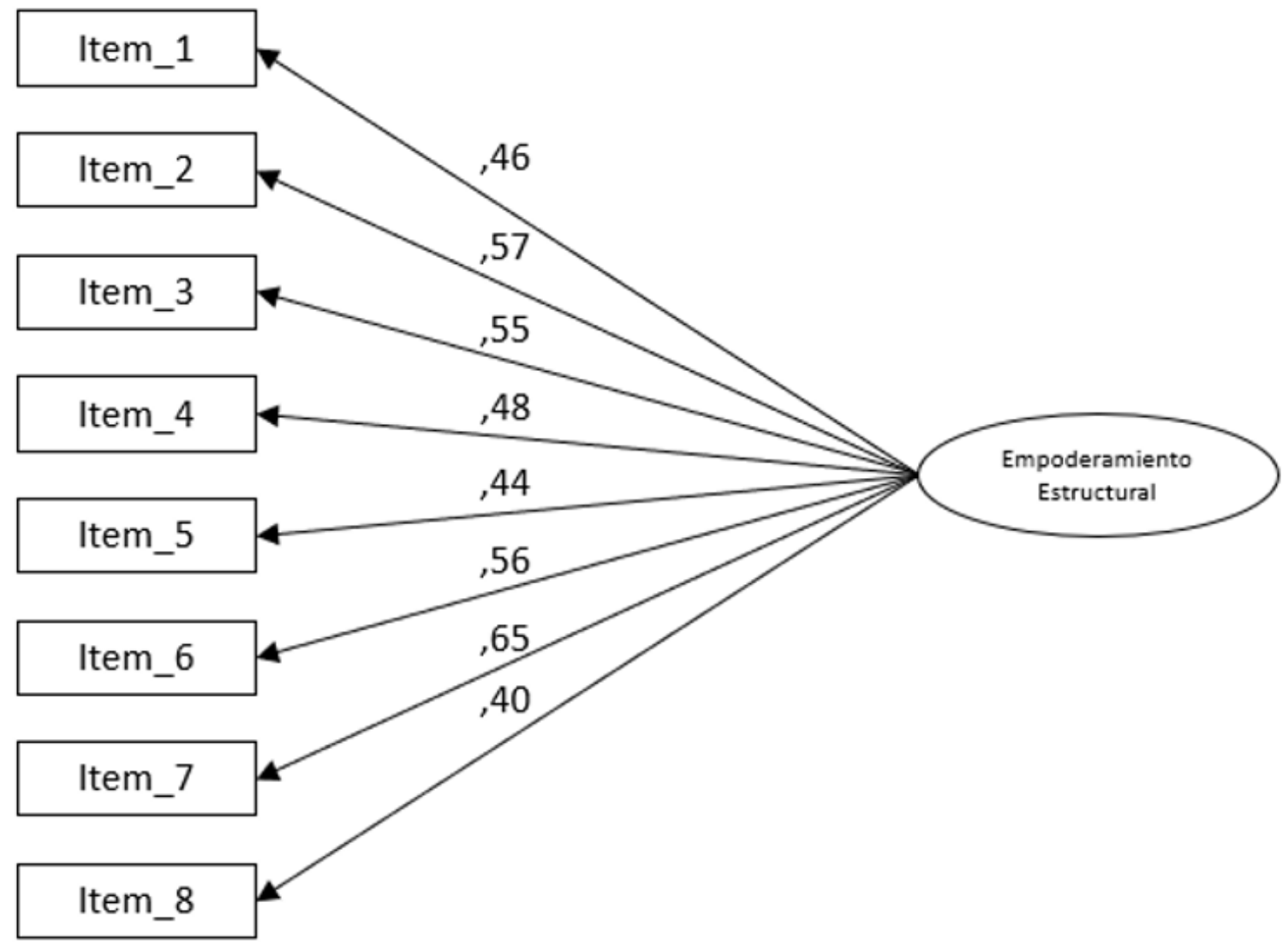

Figura 1. Modelo de ecuaciones estructurales del empoderamiento estructural.

Para el Empoderamiento psicológico el instrumento mostró un mejor ajuste al evaluar dos dimensiones $\left(X^{2}=52.89, \mathrm{DF}=24, \mathrm{p}=.0006, \mathrm{X}^{2} / \mathrm{df}=2.20, \mathrm{GFI}=.953, \mathrm{AGFI}=.913, \mathrm{CFI}=.967\right.$, RMSEA $=.082)$. Luego de eliminar los ítems que presentaron baja saturación factorial $(<.40)$,

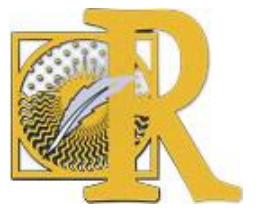

Fecha de recepción: 26-02-2020 Fecha de aceptación: 18-03-2020 
la escala quedó compuesta por 9 ítems (ver figura 2). La primera dimensión denominada Competencia (ítems $1,3,7,8,12,13$ ), hace referencia a la manera en que los estudiantes adquieren las habilidades para realizar su trabajo académico de forma independiente, usando su capacidad y conocimientos adquiridos. La segunda dimensión denominada Impacto (ítems $4,6,11)$, hace referencia a la manera como los estudiantes perciben que ejercen un efecto positivo en su entorno académico. Se encontró un nivel satisfactorio de homogeneidad medido mediante el coeficiente de fiabilidad compuesta: Competencia (.722) e Impacto (.766). Con respecto al coeficiente Alfa de Crombach se encontraron valores de .662 y .738 respectivamente.

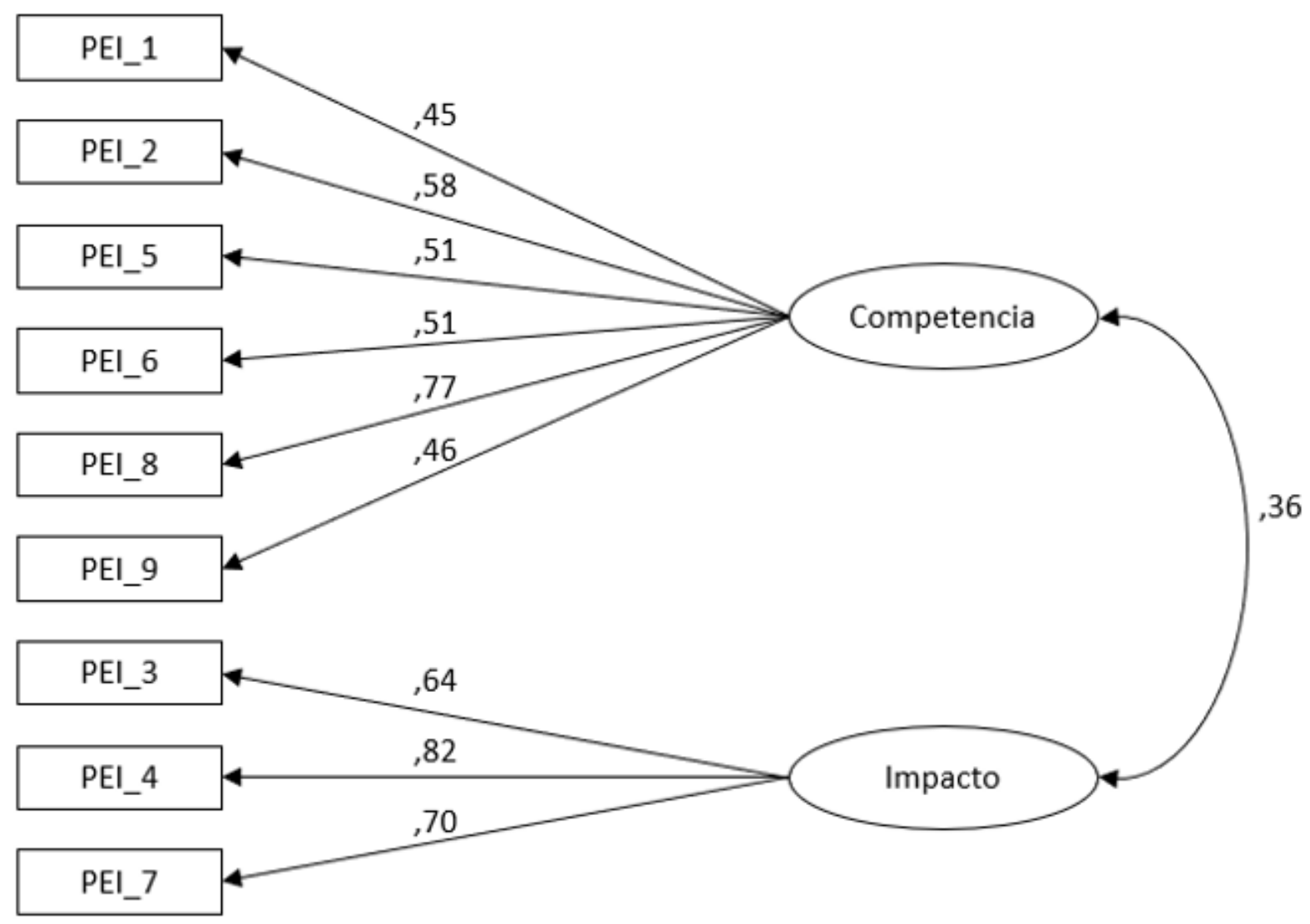

Figura 2. Modelo de ecuaciones estructurales del empoderamiento psicológico.

Previo al análisis de la hipótesis, se descartó la presencia de colinealidad entre las variables $\left(\mathrm{R}^{2}>.90\right)$. El análisis de la asimetría y curtosis resultó satisfactorio, ya que todos los valores se ubicaron entre -1 y 1 (George \& Mallery, 2010). No se detectaron casos con desviaciones mayores a 3.26 respecto de la media en alguna o más de las variables (Tabachnick \& Fidell, 2001).

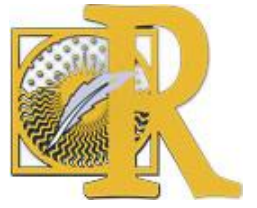

Fecha de recepción: 26-02-2020 Fecha de aceptación: 18-03-2020 


\section{INTERNATIONAL JOURNAL OF EDUCATIONAL \\ RESEARCH AND INNOVATION \\ REVISTA INTERNACIONAL DE INVESTIGACIÓN

Luego de calcular los puntajes totales de cada variable, en la Tabla 1 se presentan las correlaciones, los estadísticos descriptivos y los valores de confiabilidad de los factores representantes del empoderamiento estructural, del empoderamiento psicológico y de la autoeficacia. Se observan correlaciones significativas entre todas las variables del estudio.

Tabla 1.

Resumen de las correlaciones, media, desvío estándar y valores de confiabilidad para las variables bajo estudio.

\begin{tabular}{lllllllll} 
& 2 & 3 & 4 & $\mathrm{M}$ & $\mathrm{DE}$ & Min & Max & $\alpha$ \\
\hline 1. Autoeficacia & $.275^{*}$ & $.449^{*}$ & $.222^{*}$ & 2.95 & .51 & 1.33 & 4.00 & .77 \\
2. Emp. Estructural & & $.398^{*}$ & $.243^{*}$ & 3.82 & .55 & 2.25 & 5.00 & .67 \\
3. Competencia & & & $.262^{*}$ & 3.91 & .58 & 1.83 & 5.00 & .66 \\
4. Impacto & & & & 3.44 & .85 & 1.00 & 5.00 & .74 \\
\hline
\end{tabular}

${ }^{*} \mathrm{p}<.001$

Dadas las correlaciones obtenidas, se pretendió conocer si el empoderamiento psicológico actuaba como variable mediadora entre la relación del empoderamiento estructural y la autoeficacia académica. Se utilizó el método de máxima verosimilitud robusto para la estimación del modelo y se calcularon los indicadores más frecuentes para el diagnóstico de bondad de ajuste.

El empoderamiento académico (estructural y psicológico) explicó aproximadamente el 10\% de la varianza de la autoeficacia académica (figura 3). Los estadísticos de bondad de ajuste y medidas de error presentaron valores dentro de lo sugerido por la bibliografía para indicar un buen ajuste (Bentler, 1990) (tabla 2). El modelo mostró que el empoderamiento psicológico presenta un efecto mediador parcial en la relación del empoderamiento estructural con la autoeficacia académica.

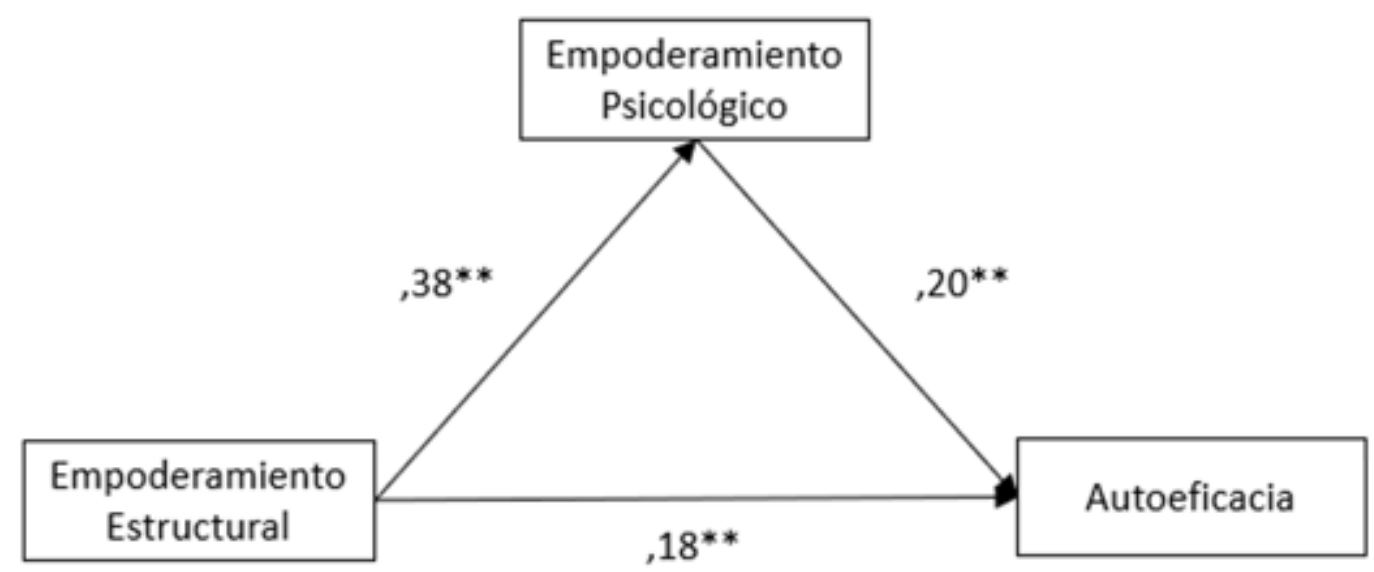

Figura 3. Coeficientes estandarizados para el modelo de ecuaciones estructurales de autoeficacia académica.

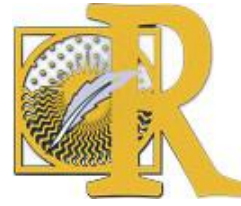

Fecha de recepción: 26-02-2020 Fecha de aceptación: 18-03-2020 
Tabla 2.

Indicadores de ajuste del modelo de autoeficacia.

\begin{tabular}{ccccccccc}
\hline Modelo & $\mathrm{X}^{2}$ & Df & $\mathrm{p}$ & CMIN/DF & GFI & CFI & RMSEA & $\begin{array}{c}\text { RMR } \\
\text { estandarizado }\end{array}$ \\
\hline 1 & 1.29 & 1 & .2637 & 1.29 & .998 & .999 & .028 & .012 \\
\hline
\end{tabular}

\section{Discusión y conclusiones.}

El propósito principal del trabajo fue evaluar la relación entre el empoderamiento académico y la autoeficacia de los estudiantes universitarios. Para ello, en primer lugar, se planteó adaptar las escalas de empoderamiento organizacional al ámbito educativo.

El empoderamiento estructural académico es entendido como la manera en que los docentes, directivos, e institución en general, brindan las condiciones y los recursos que favorecen el aprendizaje de los estudiantes. El modelo original (Laschinger, Finegan, Shamian, \& Wilk, 2004) presentaba cuatro dimensiones (información, apoyo, recursos y oportunidades) representadas por doce ítems, tres en cada una de ellas. Luego de realizar los análisis psicométricos se encontró un modelo unidimensional con mayor precisión y validez para explicar este constructo. El modelo resultante quedó compuesto con ítems de cada una de las dimensiones originales representando la totalidad de los contenidos propuestos para el constructo. Los resultados indicaron un puntaje moderadamente alto de esta variable, indicando que los estudiantes valoraban con un nivel bastante satisfactorio las condiciones óptimas de aprendizaje. Para el éxito académico es necesario que los estudiantes posean los recursos, las oportunidades y el apoyo de parte de sus docentes y directivos.

Desde esta perspectiva las instituciones deben ofrecer la contención necesaria que los educandos precisan. Más que un listado de conocimientos necesarios para la adquisición de una determinada competencia, se debe introducir a los estudiantes en un aprendizaje continuo y profundo del desarrollo en sociedad.

En el caso del empoderamiento psicológico, el modelo original (Spreitzer, 1995) estaba compuesto por cuatro dimensiones (Competencia, Autonomía, Impacto y Significado). Luego de realizar los análisis psicométricos, la versión final quedó conformada por dos dimensiones (Competencia e Impacto).

Con respecto a la dimensión competencia, los estudiantes manifestaron un nivel bastante satisfactorio, indicando que se perciben competentes en los saberes adquiridos en el cursado. Por otra parte, la dimensión impacto presentó un puntaje equivalente a un nivel de suficiente, lo que destaca la necesidad de favorecer o generar más situaciones para que el estudiante se desarrolle en esta área. El componente social es importante para el aprendizaje exitoso, de esta manera el impacto en su grupo es un aspecto necesario para valorar cómo su desempeño es considerado por quienes lo rodean. La dimensión Significado no alcanzó el nivel psicométrico adecuado, si bien en los análisis preliminares los ítems se agrupaban en un factor común, el nivel de homogeneidad de las medidas era insuficiente para ser considerado. Futuros estudios deberían indagar más sobre esta dimensión.

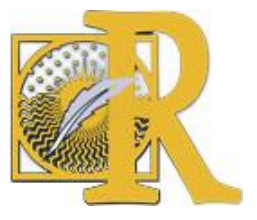

Fecha de recepción: 26-02-2020 Fecha de aceptación: 18-03-2020 
Los análisis de la autoeficacia en la muestra señalaron que los estudiantes presentaban un nivel moderadamente alto, indicando que se percibían como capaces y preparados para alcanzar el éxito académico.

Con respecto a los análisis entre las variables, se ha observado una relación significativamente positiva entre el Empoderamiento Estructural y el Psicológico, tal y como se afirmaba en el modelo propuesto (Jáimez, 2013). En la medida en que se ofrecen las condiciones y recursos necesarios a los estudiantes, se obtiene una mayor percepción de impacto y competencia hacia su aprendizaje. Se entiende por tanto que, a mayor percepción de Empoderamiento Estructural, mayor es la percepción de Empoderamiento Psicológico.

El modelo muestra que, a mayor Empoderamiento académico, mayor resulta la percepción de Autoeficacia. Por su parte, se observa el rol mediador del Empoderamiento Psicológico, puesto que el efecto del Empoderamiento Estructural sobre la Autoeficacia se potencia por su mediación. Por ende, conforme aumenta la disposición de condiciones y recursos a los estudiantes, se incrementa su percepción de competencia y por lo tanto de Autoeficacia.

Se podría inferir que, a mayor Empoderamiento Estructural y Psicológico, mayor resulta la percepción de Autoeficacia, lo que implicaría que, si un estudiante universitario percibe que la institución asume su función educativa apropiadamente, proveyendo las condiciones y recursos necesarios para desarrollar las competencias planteadas, se apreciaría con mayor nivel de autoeficacia.

De acuerdo con Baron y Kenny (1986), se deben cumplir tres condiciones para analizar el posible papel mediador de una variable, como en este caso, el Empoderamiento Psicológico. La primera es que la variable independiente (Empoderamiento Estructural) influya en la variable mediadora (Empoderamiento Psicológico); en segundo lugar, que la variable mediadora se relacione con la variable dependiente (Autoeficacia); en tercer lugar, que la variable independiente se relacione significativamente con la variable dependiente y que, una vez introducida la variable mediadora, esta relación pierda, parcial o totalmente, su efecto. Por tanto, en esta investigación se ha mostrado que el Empoderamiento Psicológico desempeña un papel mediador entre el Empoderamiento Estructural y la Autoeficacia.

Las conclusiones muestran consistencia con otras afirmaciones, como por ejemplo las de Román-Calderón et al. (2016), quienes identificaron el efecto del apoyo organizacional percibido sobre la participación en actividades planeadas por la organización y los comportamientos empoderados, lo que hace referencia a la modificación, cambios o mejoras en la manera de realizar las tareas, mediado por el empoderamiento psicológico. En este sentido, y por transferencia al público sujeto del estudio, los resultados presentados ayudan a sostener la necesidad de brindar apoyo a los estudiantes. Dicho apoyo favorece que los estudiantes desarrollen y se perciban más competentes en el proceso formativo y, consecuentemente, más autoeficaces.

En las estructuras organizacionales empoderadoras, los líderes tienen la posibilidad de aumentar la autoeficacia de sus miembros y el control que éstos tienen sobre su ambiente de trabajo (Xue, Bradley, Liang, 2011). Siguiendo esta propuesta se observa que, bajo condiciones favorables, las potencialidades de los estudiantes se ven estimuladas y desarrolladas y las habilidades pueden ser usadas bajo sus propios términos a fin de alcanzar metas claras de aprendizaje.

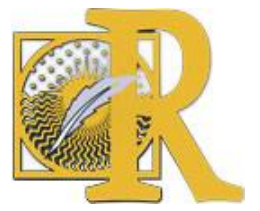

Fecha de recepción: 26-02-2020 Fecha de aceptación: 18-03-2020

Cecilia-Tumino, M., Mauricio-Quinde, J., Noemí-Casali, L. \& Raquel-Valega, M. (2020). Autoeficacia en

estudiantes universitarios: el rol del empoderamiento académico

International Journal of Educational Research and Innovation (IJERI), 14, 211-224

ISSN: 2386-4303 DOI https://doi.org/10.46661/ijeri.4618 


\section{Referencias}

- Bandura, A. (1986). Social foundations of thought and action: A social cognitive theory. Englewood Cliffs, NJ: Prentice-Hall.

- Bandura, A. (1987). Pensamiento y acción. Fundamentos sociales. Barcelona: Martínez Roca.

- Baron, R. M. y Kenny, D. A. (1986). The moderator-mediator variable distinction in social psychological research: Conceptual, strategic and statistical considerations. Journal of Personality and Social Psychology, 51, 1173-1182. Recuperado de https://www2.psych.ubc.ca/ schaller/528Readings/BaronKenny1986.pdf

- Barraza, A. (2010). Validación del inventario de expectativas de autoeficacia académica en tres muestras secuenciales e independientes. CPU-e. Revista de Investigación Educativa, 10. Recuperado de http://www.uv.mx/cpue/num10/inves/barraza validacion.html

- Bentler, P. M. (1990). Comparative fit indexes in structural models. Psychological Bulletin, 107(2), 238-246. doi:10.1037/0033-2909.107.2.238

- Blanco Vega, Humberto, Ornelas Contreras, Martha, Aguirre Chávez, Juan Francisco, \& Guedea Delgado, Julio César. (2012). Autoeficacia percibida en conductas académicas: diferencias entre hombres y mujeres. Revista mexicana de investigación educativa, 17(53), 557-571. Recuperado de http://www.scielo.org.mx /scielo.php?script=sci_arttext\&pid=S1405-66662012000200011\&lng=es\&tIng=es

- Canto y Rodríguez, J. E. (1998). Autoeficacia y educación. Educación y Ciencia, 2(4), 45 - 53. Recuperado de http://educacionyciencia.org/index.php/educacionyciencia /article/download/142/pdf

- Cavazos Arroyo, J. y Encinas Orozco, F. C. (2016). Influencia del engagement académico en la lealtad de estudiantes de posgrado: un abordaje a través de un modelo de ecuaciones estructurales. Estudios Gerenciales, 32(140), 228-238. Recuperado de https://dx.doi.org/10.1016/j.estger.2016.07.001

- Chen, H. F. and Chen, Y. C. (2008). The Impact of Work Redesign and Psychological Empowerment on Organizational Commitment in a Changing Environment: An Example from Taiwan's State-Owned Enterprises. Public Personnel Management, 37(3), 279 - 302. doi:10.1177/009102600803700302

- Dominguez, S., Villegas, G., Yauri, C., Mattos E. \& Ramírez F. (2012). Propiedades psicométricas de una escala de autoeficacia para situaciones académicas en estudiantes universitarios peruanos. Revista de Psicología-Universidad Católica San Pablo, 2(1), 2739. Recuperado de http://35.161.26.73/index.php/psicologia /article/view/8/7

- Gutiérrez-García, A. G. y Landeros-Velázquez, M. G. (2017). Evaluación de funciones ejecutivas en estudiantes universitarios con niveles de autoeficacia percibida baja. Revista Electrónica de Psicología Iztacala, 20(2), 397-426. Recuperado de https://www.iztacala.unam.mx/carreras/psicologia/psiclin/vol20num2/Vol20No2Art1.pdf

- George, D., \& Mallery, M. (2010). SPSS for Windows Step by Step: A Simple Guide and Reference, 17.0 update (10a ed.) Boston: Pearson.

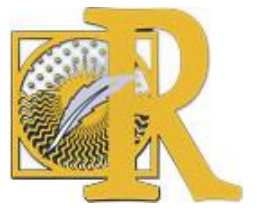

Fecha de recepción: 26-02-2020 Fecha de aceptación: 18-03-2020 
- Jáimez Román, M. J. y Bretones, F. D. (2013). Spanish Adaptation of the Structural Empowerment Scale. Spanish Journal of Psychology, 16(15), 1-7. doi:10.1017/sjp.2013.14

- Jáimez Román, M.J. (2013). Organizaciones saludables: el papel del empoderamiento organizacional. Granada: Universidad de Granada. Recuperado de http://hdl.handle.net/10481/23761

- Jöreskog, K. G., \& Sörbon, D. (2019). LISREL 10: user's reference guide. Chicago: Scientific Software International.

- Kline, R. B. (1998). Methodology in the social sciences. Principles and practice of structural equation modeling. Guilford Press.

- Laschinger, H. K. S., Finegan, J. E., Shamian, J., \& Wilk, P. (2004). A longitudinal analysis of the impact of workplace empowerment on work satisfaction. Journal of Organizational Behavior, 25(4), 527-545. doi: 10.1002/job.256

- Magendzo, A. (2003). Pedagogía crítica y educación en derechos humanos. Paulo Freire. Revista de Pedagogía Crítica, 2(2), 19-27. Recuperado de http://bibliotecadigital .academia.cl/bitstream/handle/123456789/1624/019-027.pdf?sequence $=1$

- Pajares (2002). Self-efficacy beliefs in academic contexts: An outline. Recuperado de http://www.uky.edu/ eushe2/Pajares/efftalk.html

- Palenzuela, D. L. (1983). Construcción y validación de una escala de autoeficacia percibida específica de situaciones académicas. Análisis y Modificación de Conducta, 9(21), 185219. Recuperado de https://dialnet.unirioja.es/descarga/articulo/7101317.pdf

- Pérez, F. (2018). Autoeficacia general, académica y rendimiento académico en estudiantes universitarios (Doctoral dissertation, Universidad Argentina de la Empresa). Recuperado de https://repositorio.uade.edu.ar/xmlui/bitstream/handle/123456789/7929/PEREZ\%20F.TIF.pdf? sequence $=3$ \&is Allowed $=y$

- Román-Calderón, J. P., Krikorian, A., Ruiz, C. F., \& Gaviria, A. B. (2016). Apoyo organizacional y empoderamiento como antecedentes de comportamientos empoderados y participación de los empleados. Estudios Gerenciales, 32(139), 154-161. doi:10.1016/j.estger.2016.03.002

- Sánchez, A. (2002). Dispositivos de empoderamiento para el desarrollo psicosocial. Universitas Psychologica, 1(2), 39-48. Recuperado de http://www.redalyc.org /articulo.oa?id=64701206

- Schunk, D. H. (1991). Self-efficacy and academic motivation. Educational Psychologist. 26(3), 207-231. doi:10.1080/00461520.1991.9653133

- Spreitzer, G. M. (1995). Psychological empowerment in the workplace: Dimensions, measurement, and validation. Academy of Management Journal, 38(5), 1442-1465. doi:10.5465/256865

- Steiger, J. H. (1990) Structural Model Evaluation and Modification: An Interval Estimation Approach. Multivariate Behavioral Research, 25(2), 173-180. doi: 10.1207/s15327906mbr2502_4

- Tabachnick, B.G. and Fidell, L.S. (2001) Using Multivariate Statistics. 4th Edition, Allyn and Bacon, Boston.

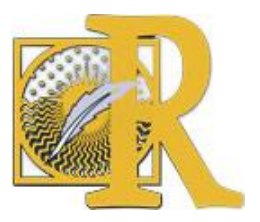

Fecha de recepción: 26-02-2020 Fecha de aceptación: 18-03-2020 
- Torres, A. (2009). La educación para el empoderamiento y sus desafíos. Sapiens. Revista Universitaria de Investigación, 10(1), 89-108. Recuperado de https://www.redalyc.org /pdf/410/41012305005.pdf

- Ventura de la Roca, D. (2012). Eficacia en estudiantes de último año de psicología clínica en relación a la práctica profesional supervisada. Tese de licenciatura da Universidad Rafael Landívar, Facultad de Humanidades, Guatemala. Recuperado de http://biblio3.url.edu.gt/Tesis/2012/05/42 /De-La-Roca-David.pdf

- Wall, L. (2012). An Exploratory Study of Teacher Empowerment and Technical Education in Kentucky. Dissertations, 38, 11-15. Recuperado de http://digitalcommons.wku.edu $\underline{\text { diss } / 38}$

- Xue, Y., Bradley, J. and Liang, H. (2011). Team climate, empowering leadership, and knowledge sharing. Journal of Knowledge Management, 15(2), 299-312. https://doi.org/10.1108/13673271111119709

- Zambrano, C. y Quinde, J. (2015). Satisfacción Laboral y Empowerment en Médicos y Enfermeros. Tesis de grado no publicada. Universidad Adventista del Plata, Entre Ríos, Argentina. 


\section{Anexos.}

\section{Inventario Académico de Empoderamiento Psicológico}

A continuación, indique el grado en el que cada una de las siguientes condiciones se da en su vida estudiantil. Para ello, escriba el número correspondiente al final de cada frase teniendo en cuenta que 1 es "poco/a" y 5 "mucho/a"

$\begin{array}{ccccc}1 & 2 & 3 & 4 & 5 \\ \text { Poco } & \text { Regular } & \text { Suficiente } & \text { Bastante } & \text { Mucho }\end{array}$

1. Tengo confianza en mi capacidad para hacer las tareas académicas que se me asignan

2. Tengo autonomía para decidir como estudiar

3. Mi influencia sobre lo que ocurre en mi grupo de estudios es alta

4. Tengo mucho control sobre lo que sucede en mi grupo de estudios

5. Puedo decidir por mí mismo/a cómo complementar mi formación profesional

6. Tengo la oportunidad de expresar mi pensamiento de forma libre y espontanea

7. Tengo bastante influencia sobre lo que ocurre en mi grupo de estudios

8. Estoy seguro de mí mismo/a acerca de mis capacidades para realizar las actividades académicas

9. Tengo la oportunidad de utilizar mi iniciativa personal para llevar a cabo mis estudios

\section{Cuestionario de Condiciones Efectivas de Aprendizaje}

A continuación, indica el grado en el que cada una de las siguientes características se da en tu lugar de estudios. Para ello, escribe el número correspondiente al final de cada frase teniendo en cuenta que 1 es "poco/a" y 5 "mucho/a".

$\begin{array}{ccccc}1 & 2 & 3 & 4 & 5 \\ \text { Poco } & \text { Regular } & \text { Suficiente } & \text { Bastante } & \text { Mucho }\end{array}$

¿Qué características consideras que tiene tu carrera actual?"

1. Estudiar es estimulante

2. Desarrollo de tareas que emplean todas mis habilidades y conocimientos

3. Información sobre el estado actual de mi carrera

4. Información sobre los valores que promueve la carrera

5. Información sobre las competencias desarrolladas durante la carrera

6. Información específica sobre las cosas que hago bien

7. Consejos útiles o sugerencias sobre la resolución de problemas

8. Tiempo disponible de preparación para los exámenes 